\title{
Molecular detection of Neorickettsia risticii in Brazilian free-tailed bats (Tadarida brasiliensis) from Buenos Aires, Argentina ${ }^{1}$
}

INDEX TERMS: Neorickettsia, Potomac Horse Fever, bats, Argentina

\section{INTRODUCTION}

The order Rickettsiales comprise a group of agents that are obligate intracellular gram-negative bacteria transmitted by vectors (mainly arthropods) and include the families Rickettsiaceae and Anaplasmataceae (Dumler et al. 2001). Neorickettsia risticii (formerly Ehrlichia risticii), Anaplasmataceae family, is the causative agent of Potomac Horse Fever (also known as Equine Monocytic Ehrlichiosis), a severe febrile disease affecting horses, typically found in endemic countries during the warmest months, especially from mid to late summer (Wilson et al. 2006). In the environment, $N$. risticii infects trematodes from the genus Acanthatrium and Lecithodendrium. Although the lifecycles of these trematodes are not very clear, both are known to involve several stages that range from free-living cercaria to forms that infect invertebrates, such as the miracidia and sporocysts (that infect freshwater snails), and the metacercaria (that infect aquatic insects). Adult forms of these trematodes develop in the intestine of insectivorous vertebrates such as bats and birds (Wilson et al. 2006, Lin et

\footnotetext{
${ }^{1}$ Received on September 26, 2012.

Accepted for publication on January 4, 2013.

${ }^{2}$ Sección Serología y Pruebas Biológicas, División Inmunología y Diagnóstico, Instituto de Zoonosis Luis Pasteur, Av. Díaz Vélez 4821, Ciudad Autónoma de Buenos Aires C1405DCD, Argentina. *Corresponding author: gcicuttin@gmail.com
}

al. 2009). It has been suggested that certain species of bats and birds (swallows) may act as wild reservoirs of $N$. risticii (Pusterla et al. 2003, Gibson et al. 2005).

T. brasiliensis are insectivorous and migratory bat whose range extends from Southern USA to Argentina and Chile. It is a common and abundant species that tend to form large colonies in major cities and urban centers (Barquez, Braun \& Mares 1999, Nabte et al. 2011). In Argentina, trematodes of the genus Lecithodendrium have been previously described to infect T. brasiliensis (Lunaschi \& Notarnicola 2010).

The aim of the present study was to detect genus Ehrlichia, Anaplasma and Neorickettsia (Anaplasmataceae family) and Rickettsia, in bats from Buenos Aires city, Argentina.

\section{MATERIALS AND METHODS}

In 2011, a total of 30 Brazilian free-tailed bats (Tadarida brasiliensis) from urban areas of Buenos Aires city were obtained. Bats were submitted to the Instituto de Zoonosis Luis Pasteur, Buenos Aires city, Argentina, for rabies testing.

At necropsy, samples from different tissues that are target to rickettsial pathogens (liver, spleen and lung) were collected and stored at $-70^{\circ} \mathrm{C}$ until further testing. Genomic DNA was extracted from pools of tissues by using AxyPrep ${ }^{\text {TM }}$ Multisource Genomic DNA Miniprep Kit (Axygen Biosciences, Union City, CA, USA). Polimerase chain reaction (PCR) was performed to amplify a $345 \mathrm{bp}$ fragment of the 16S rRNA gene for genus Ehrlichia, Anaplasma and Neorickettsia (primers: EHR16SD; 5'-GGT_ACC_YAC_AGA_ 
AGA_AGT_CC-3' and EHR16SR; 5'-TAG_CAC_TCA TCG_TTT_ACA_ GC-3'), and other PCR to amplify a 400 bp fragment of the $23 \mathrm{~S}-5 \bar{S}$ intergenic spacer for the genus Rickettsia (primers: RCK/23-5-F; 5'-GAT_AGG_TCR GRT_GTG_GAA_GCA_C-3' and RCK/23-5-R; 5' TCG_GGA_YGG_GAT_CGT GTG_TTT_C-3'), according to methods previously described (Parola et al. 2000, Jado et al. 2006). Anaplasma bovis and a plasmid of clone 4 of Rickettsia conorii (both gently provided by the Laboratorio de Espiroquetas y Patógenos Especiales, Instituto de Salud Carlos III, Spain) were used as positive controls respectively. Nuclease-free water was used as a negative control. The sensitivity threshold was $10^{3}$ organisms and 10 copies of plasmid per reaction for $16 \mathrm{~S}$ rRNA gene and 23S-5S intergenic spacer PCRs respectively.

PCR products were purified using PureLink ${ }^{\mathrm{TM}}$ Quick Gel Extraction and PCR Purification Combo Kit (Invitrogen-Life Technologies, Carlsbad, CA, USA) and sequenced with a 3500 Genetic Analyzer sequencer (Applied Biosystems, Foster City, CA, USA) at the Service of Neuroviruses, National Institute of Infectious Diseases (ANLIS Dr. Carlos G. Malbrán, Buenos Aires city, Argentina).

Sequences obtained were compared with sequences available at the GenBank database by using BLAST tool (www.ncbi.nlm. nih.gov/blast). A phylogenetic analysis was conducted with the Tamura-Nei model using MEGA version 5 (Tamura et al. 2011). Gaps were excluded in the pairwise comparison, and support for the topology was tested by bootstrapping over 1,000 replications.

\section{RESULTS}

Three bats (10\%) were positive for the 16S rRNA gene PCR (genus Ehrlichia, Anaplasma and Neorickettsia), whereas all samples were negative for Rickettsia. All positive controls showed bands of appropriate sizes, whereas negative controls yielded no products, confirming lack of amplicon contamination.

All 16S rRNA gene PCR products were sequenced and showed $100 \%$ identity with each other and $99.67 \%$ with $N$. risticii Illinois strain (Gen-Bank accession no. CP001431). Their phylogenetic relationships are shown in the Figure 1. A representative sequence was deposited in the GenBank database under the accession number: JX125043.

\section{DISCUSSION}

To our knowledge, this is the first detection of Neorickettsia risticii in Argentina and the first to be described in bats of the species Tadarida brasiliensis.

Since its first detection in 1979 in the United States, $N$. risticii has been reported in Canada, Brazil and Uruguay, either in horses, bats and swallows, as well as in snails and aquatic insects (Dutra et al. 2001, Coimbra et al. 2005, 2006, Lin et al. 2009). The role of insectivorous vertebrates in the life cycle of $N$. risticii has yet to be elucidated. Bats (and swallows) are definitive hosts for adult trematodes, which may become infected with $N$. risticii and transmit the bacterium through different lifecycle stages. Remarkably, the detection of $N$. risticii in several internal organs of bats may suggest a potential role as reservoirs of infection (Pusterla et al. 2003, Gibson et al. 2005).

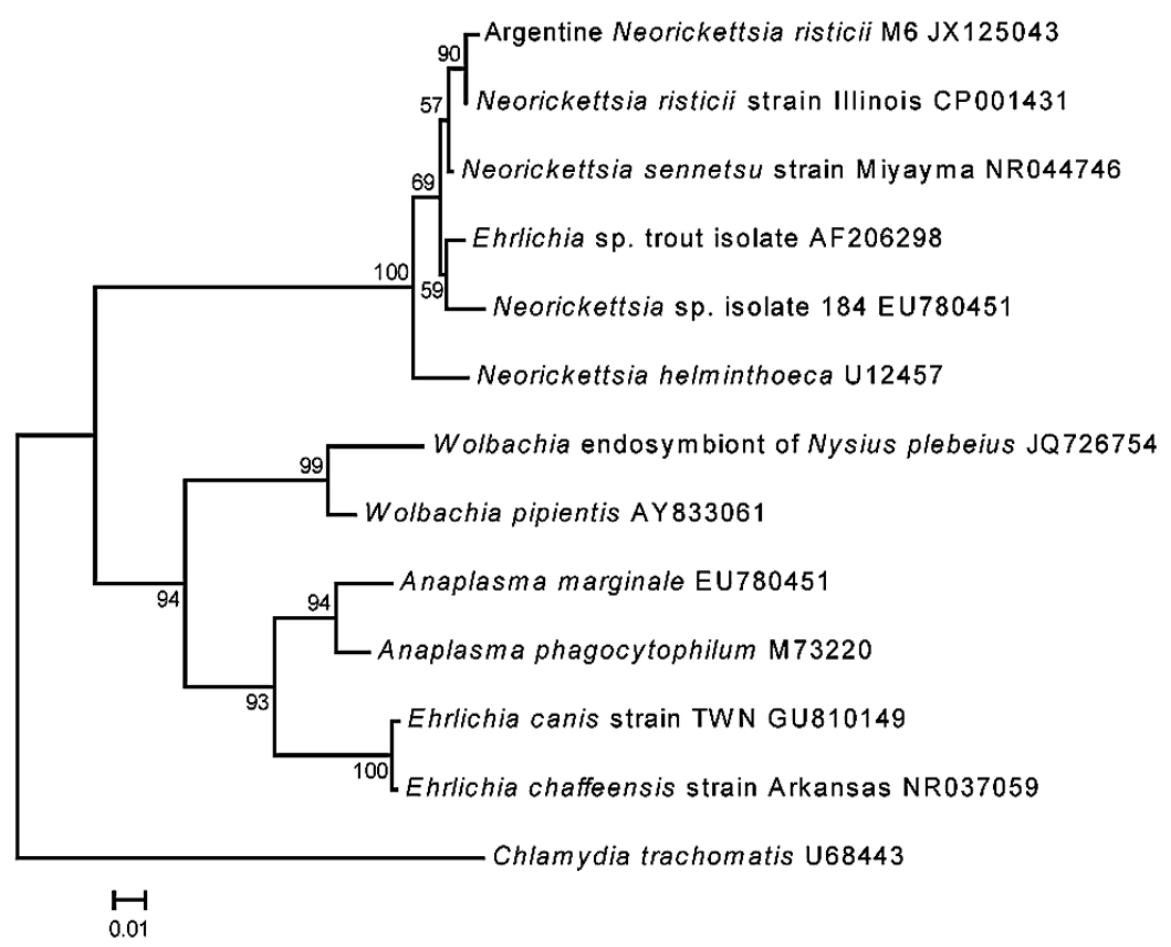

Fig.1. Phylogenetic relationship between Argentine Neorickettsia risticii M6 strain and other strains of Anaplasmataceae family based on partial sequence of the 16S rRNA gene (305 bp). Numbers above internal nodes indicate the percentage of 1,000 bootstrap replicates that supported the branch. All bootstrap values are included for clades that were consistently observed using the phylogenetic methods applied (maximum parsimony, minimum evolution, maximum likelihood and neighbor-joining trees). The neighbor-joining (NJ) tree is shown. Chlamydia trachomatis sequence was used as an outgroup. GenBank sequence accession numbers are provided. 
Previous studies in other species of bats reported from 11.1 to $60 \%$ positive for $N$. risticii by PCR from liver, spleen and blood (Pusterla et al. 2003, Gibson et al. 2005). In comparison, we detected $10 \%(3 / 30)$ infected bats using pools of organs. The discrepancies of results could be due to number of samples analyzed, methodology used and different bats species studied.

While the importance of $N$. risticii infection on the bat population is unknown (Gibson et al. 2005), in horses it can cause severe illness, characterized by an acute enterocolitis syndrome, as well as abortion in pregnant mares. Furthermore, many horses that become clinically sick can develop laminitis. The lethality rate ranges from 5.0 to $46.1 \%$. Early recognition is the key to minimizing the impact of the disease (Dutra et al. 2001, Coimbra et al. 2006). No zoonotic risk is known. The impact of infection in bats is beyond the objectives of this study.

Given the potential impact on veterinary medicine, the confirmation of $N$. risticii in Argentina is important to increase awareness of this agent in the country, and specially to help improve notifications of suspected cases in horses to the national disease surveillance system. Additional studies are necessary to understand the ecology and transmission of $N$. risticii among vertebrates and invertebrates in the region.

Acknowledgment.- To Alberto A. Pérez (Predict Amazon regional coordinator - Wildlife Conservation Society).

\section{REFERENCES}

Barquez R.M., Braun J.K. \& Mares M.A. 1999. The bats of Argentina. Special Publications of the Museum 42. Texas Tech University Press, Lubbock. 275p.

Coimbra H.S., Schuch L.F.D., Veitenheimer-Mendes I.L. \& Meireles M.C.A. 2005. Neorickettsia (Ehrlichia) risticii no sul do Brasil: Heleobia spp. (Mollusca: Hydrobilidae) e Parapleurolophocecous cercariae (Trematoda: Digenea) como possíveis vetores. Arqs Inst. Biológico, São Paulo, 72(3):325-329.

Coimbra H.S., Fernandes C.G., Soares M.P., Meireles M.C.A., Radamés R. \& Schuch L.F.D. 2006. Equine monocytic Ehrlichiosis in Rio Grande do Sul: clinical, pathological and epidemiological aspects. Pesq. Vet. Bras. 26(2):97-101.
Dumler J.S., Barbet A.F., Bekker C.P.J., Dasch G.A., Palmer G.H., Ray S.C., Rikihisa Y. \& Rurangirwa F.R. 2001. Reorganization of genera in the families Rickettsiaceae and Anaplasmataceae in the order Rickettsiales: unification of some species of Ehrlichia with Anaplasma, Cowdria with Ehrlichia and Ehrlichia with Neorickettsia, descriptions of six new species combinations and designation of Ehrlichia equi and 'HGE agent' as subjective synonyms of Ehrlichia phagocytophila. Int. J. Syst. Evol. Microbiol. 51:2145-2165.

Dutra F., Schuch L.D., Delucchi E., Curcio B.R., Coimbra H., Raffi M.B., Dellagostin 0. \& Riet-Correa F. 2001. Equine monocytic Ehrlichiosis (Potomac horse fever) in horses in Uruguay and southern Brazil. J. Vet. Diagn. Invest. 13:433-437.

Gibson K.E., Rikihisa Y., Zhang C. \& Martin C. 2005. Neorickettsia risticii is vertically transmitted in the trematode Acanthatrium oregonense and horizontally transmitted to bats. Environ. Microbiol. 7(2):203-212.

Jado I., Escudero R., Gil H., Jiménez-Alonso M.I., Sousa R., García-Pérez A.L., Rodríguez-Vargas M., Lobo B. \& Anda P. 2006. Molecular method for identification of Rickettsia species in clinical and environmental samples. J. Clin. Microbiol. 44(12):4572-4576.

Lin M., Zhang C., Gibson K. \& Rikihisa Y. 2009. Analysis of complete genome sequence of Neorickettsia risticii: causative agent of Potomac horse fever. Nucleic Acids Res. 37(18):6076-6091.

Lunaschi L.I. \& Notarnicola J. 2010. New host records for Anenterotrematidae, Lecithodendriidae and Urotrematidae trematodes in bats from Argentina, with redescription of Anenterotrema liliputianum. Revta Mexicana Biodivers. 81:281-287.

Nabte M.J., Andrade A., Monjeau A., Hernandez J.L., Vaquero D. \& Saba S.L. 2011. Mammalia, Chiroptera, Molossidae, Tadarida brasiliensis I. Geoffroy, 1824: distribution extension. J. Species Lists and Distribution 7(2):142-143.

Parola P., Roux V., Camicas J.L., Baradji I., Brouqui P. \& Raoult D. 2000. Detection of Ehrlichiae in African ticks by polymerase chain reaction. Trans. R. Soc. Trop. Med. Hyg. 94:707-708.

Pusterla N., Johnson E.M., Chae J.S. \& Madigan J.E. 2003. Digenetic trematodes, Acanthatrium sp. and Lecithodendrium sp., as vectors of Neorickettsia risticii, the agent of Potomac horse fever. J. Helminthol. 77(4):335339.

Tamura K., Peterson D., Peterson N., Stecher G., Nei M. \& Kumar S. 2011. MEGA5: molecular evolutionary genetics analysis using maximum likelihood, evolutionary distance, and maximum parsimony methods. Mol. Biol. Evol. 28:2731-2739.

Wilson J.H., Pusterla N., Bengfort J.M. \& Arney L. 2006. Incrimination of mayflies as a vector of Potomac Horse Fever in an outbreak in Minnesota. Medicine II AAEP Proceedings 52:324-328. 